\title{
The Effects of Intense Physical Activity on Stress in Adolescents: Findings from Korea Youth Risk Behavior Web-Based Survey (2015-2017)
}

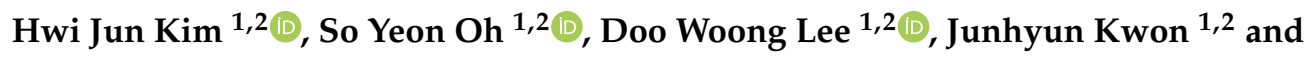 \\ Eun-Cheol Park $2,3, *$ (D) \\ 1 Department of Public Health, Graduate School, Yonsei University, Seoul 03722, Korea; \\ kimhwijun88@yuhs.ac (H.J.K.); sarahoh@yuhs.ac (S.Y.O.); doowoonglee@yuhs.ac (D.W.L.); \\ judekwon@yuhs.ac (J.K.) \\ 2 Institute of Health Services Research, Yonsei University, Seoul 03722, Korea \\ 3 Department of Preventive Medicine, Yonsei University College of Medicine, Seoul 03722, Korea \\ * Correspondence: ECPARK@yuhs.ac; Tel.: +82-2-2228-1862
}

Received: 23 April 2019; Accepted: 26 May 2019; Published: 27 May 2019

\begin{abstract}
The purpose of this study was to investigate the association between intense physical activity and stress in Korean adolescents. The study used data from the Korea Youth Risk Behavior Web-Based Survey (KYRBWS), 2015-2017, that included 170,359 responses from Korean adolescents. Intense activity and stress were measured by self-diagnosis. Additionally, the chi-square test and multiple logistic regression analyses were used. It was revealed that $78.9 \%$ of Korean adolescents were exposed to stress. Students who engaged in physical activity more than five times per week were less likely to be stressed than those who did not (boys odds ratio (OR): 0.79 , confidence interval (CI): 0.78-0.80, $p$ for trend: <0.0001; girls OR: 0.77, CI: 0.75-0.79, $p$ for trend: <0.0001). The results indicated the same tendency among both boys and girls. The results of subgroup analysis revealed that students living with relatives or in childcare facilities were more likely to experience stress if they had insufficient exercise. In addition, the results confirmed that the probability of suicidal ideation increased as the frequency of exercise decreased. This study suggests that intense physical activity in Korean adolescents has a positive effect on stress management in both boys and girls. Hence, physical activity should be encouraged and implemented for managing stress.
\end{abstract}

Keywords: intense physical activity; stress; residence type; suicidal ideation; Korea Youth Risk Behavior Web-Based Survey

\section{Introduction}

Stress is an organism's response to a stressor such as an environmental condition [1,2]. This can affect the body physically or psychologically. A Canadian expert has categorized stress into good stress and bad stress and defined these as eustress and distress, respectively. Although eustress may manifest as an immediate burden, it is a stress to develop life in the future. Distress has been defined as being able to cause anxiety, depression, and other symptoms that persist despite coping or adaptation [3-5]. Many previous studies that we refer to suggested that stress is related to mental health, such as depression and suicide, in terms of distress, and may also affect sleep, cardiovascular disease, and immune system disorders.

Adolescence represents the transition from childhood to adulthood and is a period characterized by physical, physiological, psychological, and mental maturation; thus, this period is marked by an unstable psychological state [6-8]. An unstable psychological state is caused by social factors such as 
schooling, personal appearance, reason and friendship, and career-related concerns; these factors can lead to stress and negatively affect the psychological state of adolescents $[9,10]$.

According to the "Youth Statistics" data published by the Ministry of Health and Welfare in 2007, the perceived stress level in Korean adolescents decreased from $49.9 \%$ in 2007 to $39.8 \%$ in 2017, but 4 out of 10 Korean adolescents still experience stress [11]. Therefore, continuous efforts to improve these phenomena are needed.

As a way to manage stress, governments and professional organizations worldwide offer various measures, such as psychotherapy, exercise therapy, and mental-social interventions [12-17]. The Korea Centers for Disease Control and Prevention suggest that exercise is effective in managing stress. Further, exercise is effective in improving personal health, as well as maintaining interpersonal relationships $[18,19]$. According to the results of the Korea Sports Promotion Foundation, it is suggested that about 10-20 min of intense physical activity can help manage mental health [20]. A number of previous studies have also provided supporting evidence [21,22]. However, few studies have investigated the relationship between vigorous physical activity and stress. Therefore, we focused on determining how intense physical activity might be related to stress.

\section{Materials and Methods}

\subsection{Study Participants}

We obtained data for our study population from the 2015-2017 Korea Youth Risk Behavior Web-Based Survey (KYRBWS), a cross-sectional, nationwide survey conducted by the Korea Centers for Disease Control and Prevention (KCDC) (approval number 117058). The total number of survey participants during the three years of the study was 195,847. In the database, people who responded to "how many days included over 20 min of intense physical activity during the last 7 days" were surveyed from 0 to 7 days, and non-responders were excluded from the study. Examples of vigorous physical activity in this survey included jogging, soccer, basketball, climbing, cycling, and swimming. Further, we excluded data missing from the time spent sitting and studying on weekdays $(n=25,488)$. In addition, we included the samples that had responses to questions regarding gender, age, household income, residence type, number of days of weight training per week, time spent sitting and studying on weekdays, grade, alcohol consumption, smoking, depression, and suicidal thoughts. Finally, 170,359 samples (90,041 boys and 80,418 girls) were included as representative of Korean adolescents.

\subsection{Variables}

The number of days of intense physical activity per week was the main dependent variable in this study. In the KYRBWS, the frequency of weekly intense physical activity was reported by respondents using the responses "no", "1 day", "2 days", “3 days", " 4 days", “5 days", "6 days" or "7 days" per week.

The independent variable was stress. The questionnaire asked "how often do you feel stress?". Possible answers included "feel very much", "feel a lot", "feel a little", "not feel much", "not feel at all". We classified people who responded "feel very much" and "feel a lot" into a stress group, and classified the remaining respondents into a non-stress group. Furthermore, several covariates including sociodemographic, economic, and health-related characteristics were assessed. Sociodemographic characteristics included ages of 14 (middle school, grade 1), 15 (middle school, grade 2), 16 (middle school, grade 3), 17 (high school, grade 1), 18 (high school, grade 2), and 19 (high school, grade 3). Economic characteristics included household income (low, medium-low, medium, medium-high, high), residence type (live with family, live with relatives, live in accommodation, in a dormitory, live in a childcare facility), and grade (high, medium-high, medium, medium-low, low). Health-related characteristics included the number of days of weight training per week (non-weight training, more than 5 days, 4 days, 3 days, 2 days, or 1 day per week), alcohol consumption, smoking, depression, suicidal thoughts, and time spent sitting and studying on weekdays $(<8,8 \leq n<12,12 \leq n<16,16 \leq n<20)$. 


\subsection{Statistical Analysis}

The chi-square test and multiple logistic regression analysis were used to analyze the data. The chi-square test was used to examine the significant difference in stress depending on intense physical activity. Multiple logistic regression analysis was used to determine odds ratios (ORs) and 95\% confidence intervals (CIs) after adjusting for covariates. In these subgroup analyses, we used the Cochran-Armitage Test to explore the two-sided $p$-value for trend in each of the variables and stress to relate the number of days of intense physical activity per week with stress. For all data analysis, we used SAS version 9.4 (SAS Institute, Cary, NC, USA) and the significance level was set at $p$-value $<0.05$, as well as at $p$-value for trend $<0.05$.

\section{Results}

\subsection{Study Participants}

Table 1 presents the general characteristics of study participants who analyzed the stress of boys and girls by socio-demographic factors, socio-economic status, health behavior, and other related variables (number of days of weight training, time spent sitting and studying on a weekday, grade). In the chi-square test of stress and all covariates, the $p$-value was $<0.0001$, which was significant. In this analysis, $73.2 \%$ of boys and $84.5 \%$ of girls responded that they felt stressed. We observed that girls had a lower frequency of exercise than boys. In addition, we found that the stress response rate tended to decrease with groups that exercise frequently for both boys and girls. 
Table 1. General characteristics of study observations (2015-2017).

\begin{tabular}{|c|c|c|c|c|c|c|c|c|c|c|c|c|c|c|}
\hline \multirow{3}{*}{ Variable } & \multicolumn{6}{|c|}{ Boys } & \multirow{3}{*}{$p$-Value } & \multicolumn{6}{|c|}{ Girls } & \multirow{3}{*}{$p$-Value } \\
\hline & \multicolumn{2}{|c|}{ Total } & \multicolumn{2}{|c|}{ Stress } & \multicolumn{2}{|c|}{ Non-Stress } & & \multicolumn{2}{|c|}{ Total } & \multicolumn{2}{|c|}{ Stress } & \multicolumn{2}{|c|}{ Non-Stress } & \\
\hline & $n$ & $(\%)$ & $n$ & $(\%)$ & $n$ & $(\%)$ & & $n$ & $(\%)$ & $n$ & $(\%)$ & $n$ & $(\%)$ & \\
\hline \multicolumn{7}{|c|}{ Number of days of intense physical activity per week } & $<0.0001$ & & & & & & & $<0.0001$ \\
\hline More than 5 days a week & 21,876 & 24.3 & 15,161 & 69.3 & 6715 & 30.7 & & 7065 & 8.8 & 5779 & 81.8 & 1286 & 18.2 & \\
\hline 4 days a week & 8423 & 9.4 & 6043 & 71.7 & 2380 & 28.3 & & 4004 & 5.0 & 3291 & 82.2 & 713 & 17.8 & \\
\hline 3 days a week & 16,197 & 18.0 & 11,739 & 72.5 & 4458 & 27.5 & & 10,182 & 12.7 & 8370 & 82.2 & 1812 & 17.8 & \\
\hline 2 days a week & 17,154 & 19.1 & 12,954 & 75.5 & 4200 & 24.5 & & 14,471 & 18.0 & 12,161 & 84.0 & 2310 & 16.0 & \\
\hline 1 days a week & 14,123 & 15.7 & 10,749 & 76.1 & 3374 & 23.9 & & 18,610 & 23.2 & 15,814 & 85.0 & 2796 & 15.0 & \\
\hline Non-physical activity & 12,268 & 13.6 & 9302 & 75.8 & 2966 & 24.2 & & 25,986 & 32.4 & 22,418 & 86.3 & 3568 & 13.7 & \\
\hline Age & & & & & & & $<0.0001$ & & & & & & & $<0.0001$ \\
\hline 14 (middle school 1) & 15,558 & 17.3 & 10,420 & 67.0 & 5138 & 33.0 & & 14,266 & 17.8 & 11,221 & 78.7 & 3045 & 21.3 & \\
\hline 15 (middle school 2) & 16,044 & 17.8 & 11,385 & 71.0 & 4659 & 29.0 & & 14,443 & 18.0 & 11,888 & 82.3 & 2555 & 17.7 & \\
\hline 16 (middle school 3) & 16,420 & 18.2 & 11,823 & 72.0 & 4597 & 28.0 & & 15,153 & 18.9 & 12,516 & 82.6 & 2637 & 17.4 & \\
\hline 17 (high school 1) & 14,356 & 15.9 & 10,755 & 74.9 & 3601 & 25.1 & & 12,644 & 15.7 & 10,948 & 86.6 & 1696 & 13.4 & \\
\hline 18 (high school 2) & 14,814 & 16.5 & 11,581 & 78.2 & 3233 & 21.8 & & 12,709 & 15.8 & 11,335 & 89.2 & 1374 & 10.8 & \\
\hline 19 (high school 3) & 12,849 & 14.3 & 9984 & 77.7 & 2865 & 22.3 & & 11,103 & 13.8 & 9925 & 89.4 & 1178 & 10.6 & \\
\hline \multicolumn{7}{|l|}{ Household income } & $<0.0001$ & & & & & & & $<0.0001$ \\
\hline High & 10,349 & 11.5 & 6579 & 63.6 & 3770 & 36.4 & & 5888 & 7.3 & 4325 & 73.5 & 1563 & 26.6 & \\
\hline Medium-high & 25,118 & 27.9 & 17,845 & 71.0 & 7273 & 29.0 & & 20,807 & 25.9 & 16,997 & 81.7 & 3810 & 18.3 & \\
\hline Medium & 40,329 & 44.8 & 29,882 & 74.1 & 10,447 & 25.9 & & 40,262 & 50.1 & 34,445 & 85.6 & 5817 & 14.5 & \\
\hline Medium-low & 11,335 & 12.6 & 9258 & 81.7 & 2077 & 18.3 & & 11,046 & 13.8 & 9957 & 90.1 & 1089 & 9.9 & \\
\hline Low & 2910 & 3.2 & 2384 & 81.9 & 526 & 18.1 & & 2315 & 2.9 & 2109 & 91.1 & 206 & 8.9 & \\
\hline \multicolumn{7}{|l|}{ Residence type } & $<0.0001$ & & & & & & & $<0.0001$ \\
\hline Live with family & 85,770 & 95.3 & 62,738 & 73.2 & 23,032 & 26.9 & & 77,208 & 96.1 & 65,119 & 84.3 & 12,089 & 15.7 & \\
\hline Live with relative & 857 & 1.0 & 633 & 73.9 & 224 & 26.1 & & 621 & 0.8 & 553 & 89.1 & 68 & 11.0 & \\
\hline Accommodation, dormitory & 2846 & 3.2 & 2203 & 77.4 & 643 & 22.6 & & 2131 & 2.7 & 1885 & 88.5 & 246 & 11.5 & \\
\hline Childcare facility & 568 & 0.6 & 374 & 65.9 & 194 & 34.2 & & 358 & 0.5 & 276 & 77.1 & 82 & 22.9 & \\
\hline \multicolumn{7}{|c|}{ Number of days of weight training per week } & $<0.0001$ & & & & & & & $<0.0001$ \\
\hline More than 5 days a week & 14,323 & 15.9 & 10,119 & 70.7 & 4204 & 29.4 & & 3513 & 4.4 & 2889 & 82.2 & 624 & 17.8 & \\
\hline 4 days a week & 4766 & 5.3 & 3420 & 71.8 & 1346 & 28.2 & & 1539 & 1.9 & 1252 & 81.4 & 287 & 18.7 & \\
\hline 3 days a week & 10,514 & 11.7 & 7496 & 71.3 & 3018 & 28.7 & & 4023 & 5.0 & 3298 & 82.0 & 725 & 18.0 & \\
\hline 2 days a week & 13,048 & 14.5 & 9529 & 73.0 & 3519 & 27.0 & & 6803 & 8.5 & 5615 & 82.5 & 1188 & 17.5 & \\
\hline 1 days a week & 17,112 & 19.0 & 12,596 & 73.6 & 4516 & 26.4 & & 13,859 & 17.3 & 11,460 & 82.7 & 2399 & 17.3 & \\
\hline Non-weight training & 30,278 & 33.6 & 22,788 & 75.3 & 7490 & 24.7 & & 50,581 & 63.0 & 43,319 & 85.6 & 7262 & 14.4 & \\
\hline
\end{tabular}


Table 1. Cont

\begin{tabular}{|c|c|c|c|c|c|c|c|c|c|c|c|c|c|c|}
\hline \multirow{3}{*}{ Variable } & \multicolumn{6}{|c|}{ Boys } & \multirow{3}{*}{$p$-Value } & \multicolumn{6}{|c|}{ Girls } & \multirow{3}{*}{$p$-Value } \\
\hline & \multicolumn{2}{|c|}{ Total } & \multicolumn{2}{|c|}{ Stress } & \multicolumn{2}{|c|}{ Non-Stress } & & \multicolumn{2}{|c|}{ Total } & \multicolumn{2}{|c|}{ Stress } & \multicolumn{2}{|c|}{ Non-Stress } & \\
\hline & $n$ & $(\%)$ & $n$ & $(\%)$ & $n$ & $(\%)$ & & $n$ & $(\%)$ & $n$ & $(\%)$ & $n$ & $(\%)$ & \\
\hline \multicolumn{7}{|c|}{ Time spent sitting and studying on a weekday (hours) } & $<0.0001$ & & & & & & & $<0.0001$ \\
\hline$<8$ & 45,158 & 50.2 & 32,410 & 71.8 & 12,748 & 28.2 & & 27,262 & 33.9 & 22,703 & 83.3 & 4559 & 16.7 & \\
\hline $8 \leq n<12$ & 19,429 & 21.6 & 14,061 & 72.4 & 5368 & 27.6 & & 20,195 & 25.1 & 16,902 & 83.7 & 3293 & 16.3 & \\
\hline $12 \leq n<16$ & 15,512 & 17.2 & 11,702 & 75.4 & 3810 & 24.6 & & 19,300 & 24.0 & 16,371 & 84.8 & 2929 & 15.2 & \\
\hline $16 \leq n<20$ & 9942 & 11.0 & 7775 & 78.2 & 2167 & 21.8 & & 13,561 & 16.9 & 11,857 & 87.4 & 1704 & 12.6 & \\
\hline Grade & & & & & & & $<0.0001$ & & & & & & & $<0.0001$ \\
\hline High & 12,344 & 13.7 & 8349 & 67.6 & 3995 & 32.4 & & 8259 & 10.3 & 6,430 & 77.9 & 1829 & 22.2 & \\
\hline Medium-high & 21,234 & 23.6 & 15,322 & 72.2 & 5912 & 27.8 & & 20,132 & 25.1 & 16,632 & 82.6 & 3500 & 17.4 & \\
\hline Medium & 24,807 & 27.6 & 18,022 & 72.7 & 6785 & 27.4 & & 23,508 & 29.3 & 19,858 & 84.5 & 3650 & 15.5 & \\
\hline Medium-low & 21,020 & 23.3 & 15,937 & 75.8 & 5083 & 24.2 & & 20,047 & 25.0 & 17,459 & 87.1 & 2588 & 12.9 & \\
\hline Low & 10,636 & 11.8 & 8318 & 78.2 & 2318 & 21.8 & & 8372 & 10.4 & 7,454 & 89.0 & 918 & 11.0 & \\
\hline Alcohol consumption & & & & & & & $<0.0001$ & & & & & & & $<0.0001$ \\
\hline Yes & 38,778 & 43.1 & 29,950 & 77.2 & 8828 & 22.8 & & 27,254 & 33.9 & 24,137 & 88.6 & 3117 & 11.4 & \\
\hline No & 51,263 & 56.9 & 35,998 & 70.2 & 15,265 & 29.8 & & 53,064 & 66.1 & 43,696 & 82.4 & 9368 & 17.7 & \\
\hline Smoking & & & & & & & $<0.0001$ & & & & & & & $<0.0001$ \\
\hline Yes & 19,837 & 22.0 & 15,544 & 78.4 & 4293 & 21.6 & & 6391 & 8.0 & 5732 & 89.7 & 659 & 10.3 & \\
\hline No & 70,204 & 78.0 & 50,404 & 71.8 & 19,800 & 28.2 & & 73,927 & 92.0 & 62,101 & 84.0 & 11,826 & 16.0 & \\
\hline Depression & & & & & & & $<0.0001$ & & & & & & & $<0.0001$ \\
\hline Yes & 17,592 & 19.5 & 16,102 & 91.5 & 1490 & 8.5 & & 23,307 & 29.0 & 22,372 & 96.0 & 935 & 4.0 & \\
\hline No & 72,449 & 80.5 & 49,846 & 68.8 & 22,603 & 31.2 & & 57,011 & 71.0 & 45,461 & 79.7 & 11,550 & 20.3 & \\
\hline Suicidal ideation & & & & & & & $<0.0001$ & & & & & & & $<0.0001$ \\
\hline Yes & 8227 & 9.1 & 7667 & 93.2 & 560 & 6.8 & & 11,597 & 14.4 & 11,236 & 96.9 & 361 & 3.1 & \\
\hline No & 81,814 & 90.9 & 58,281 & 71.2 & 23,533 & 28.8 & & 68,721 & 85.6 & 56,597 & 82.4 & 12,124 & 17.6 & \\
\hline Total & 90,041 & 100 & 65,948 & 73.2 & 24,093 & 26.8 & & 80,318 & 100 & 67,833 & 84.5 & 12,485 & 15.5 & \\
\hline
\end{tabular}




\subsection{Factors Associated with Physical Activity and Stress}

Using logistic regression analysis, Table 2 demonstrates the relationship between stress and the number of days of intense physical activity per week. Regarding the number of days of intense physical activity per week, groups with frequent physical activity were observed to have a tendency towards a reduced stressed odds ratio, which was significant. However, boys who engaged in physical activity one to two times per week were found to have similar levels of stress to the non-physical activity group. In addition, adolescents tended to feel more stressed as the amount of time spent sitting and studying on a weekday increased. Furthermore, the analysis found that as grades became poorer, more stress was experienced. We verified the significance of linear trends for each analysis through the $p$ for trend analysis. For both boys and girls, the $p$ value for trend was $<0.0001$, which was significant.

Table 2. Adjusted association factors associated with physical activity and stress (2015-2017).

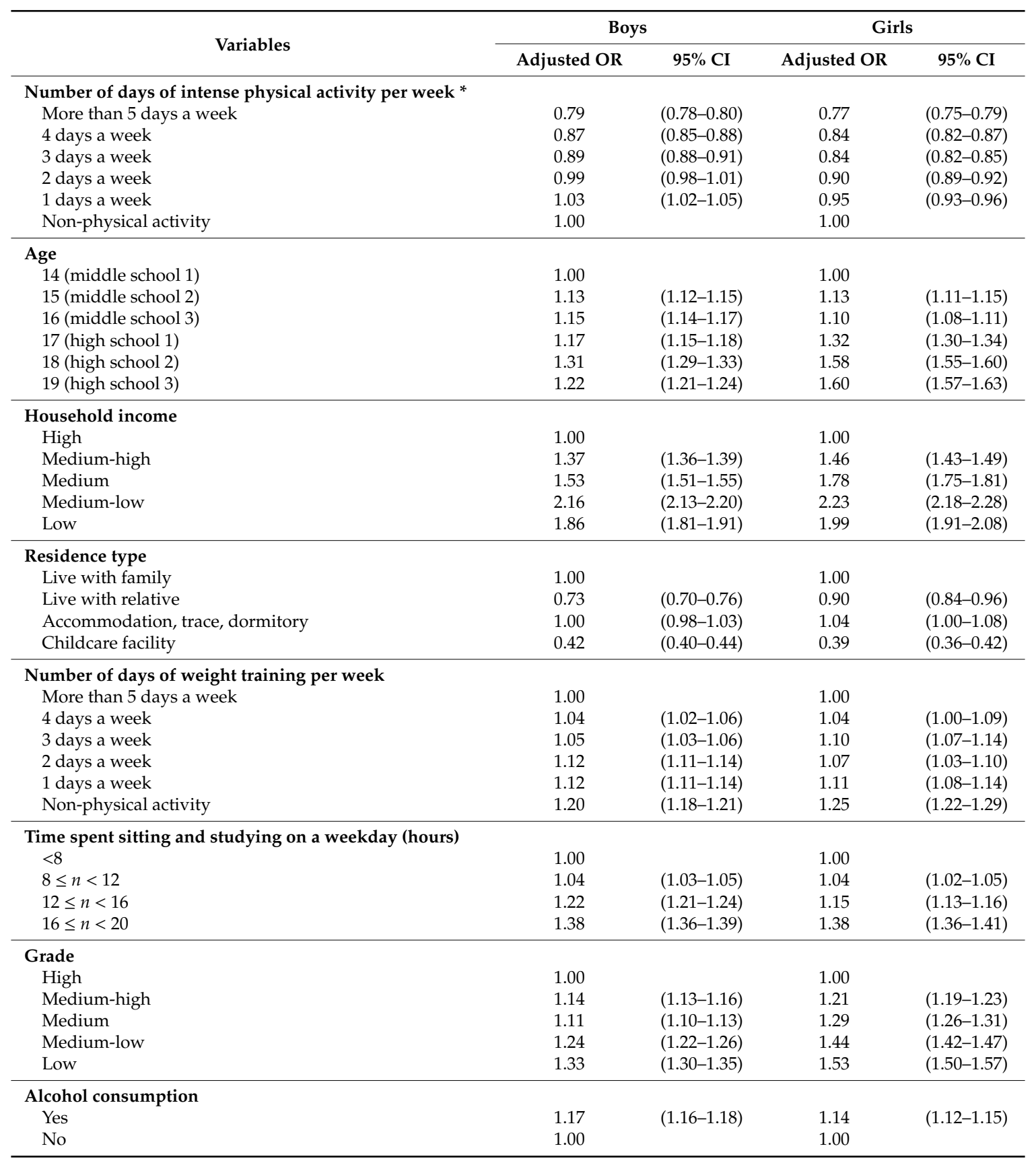


Table 2. Cont

\begin{tabular}{|c|c|c|c|c|}
\hline \multirow{2}{*}{ Variables } & \multicolumn{2}{|c|}{ Boys } & \multicolumn{2}{|c|}{ Girls } \\
\hline & Adjusted OR & $95 \%$ CI & Adjusted OR & $95 \% \mathrm{CI}$ \\
\hline \multicolumn{5}{|c|}{ Smoking } \\
\hline Yes & 1.03 & $(1.02-1.04)$ & 0.94 & $(0.91-0.96)$ \\
\hline No & 1.00 & & 1.00 & \\
\hline \multicolumn{5}{|c|}{ Depression } \\
\hline Yes & 3.70 & $(3.65-3.75)$ & 4.29 & $(4.21-4.36)$ \\
\hline No & 1.00 & & 1.00 & \\
\hline \multicolumn{5}{|c|}{ Suicidal ideation } \\
\hline Yes & 2.74 & $(2.68-2.80)$ & 3.20 & $(3.11-3.29)$ \\
\hline No & 1.00 & & 1.00 & \\
\hline
\end{tabular}

3.3. Association between Intense Physical Activity and Stress Stratified by Household Income, Residence Type, Time Spent Sitting and Studying on a Weekday, Grade, Depression, and Suicidal Ideation

Table 3 illustrates the results of subgroup analysis associating stress with intense physical activity and household income, type of residence, time spent sitting and studying on a weekday, grades, depression, and suicidal ideation. In this analysis, when the students who do not exercise even once per week are taken as the criteria, the more intense physical activity is performed in most variables, the less likely they are to be stressed.

In most subgroup analyses, frequent intense physical activity was associated with reduced stress. In particular, the analysis of girls' residence type showed that frequent intense physical activity was found to be effective for girls who lived with relatives and those who lived in childcare facility groups. Furthermore, the analysis of depression and suicidal ideation also showed that boys and girls were more likely to participate in physical activity and experience fewer mental health problems. 
Table 3. Subgroup analysis of the association between intense physical activity and stress stratified by household income, residence type, time spent and studying for a weekday, grade, depression, and suicidal ideation.

\begin{tabular}{|c|c|c|c|c|c|c|c|c|c|c|c|c|}
\hline \multirow{3}{*}{ Variable } & \multicolumn{11}{|c|}{ Number of Days of Intense Physical Activity per Week } & \multirow{3}{*}{$\begin{array}{l}p \text {-Value } \\
\text { for Trend }\end{array}$} \\
\hline & \multicolumn{2}{|c|}{ More than 5 Days a Week } & \multicolumn{2}{|c|}{4 Days } & \multicolumn{2}{|c|}{3 Days } & \multicolumn{2}{|c|}{2 Days } & \multicolumn{2}{|c|}{1 Day } & \multirow{2}{*}{$\begin{array}{c}\begin{array}{c}\text { Non-Physical } \\
\text { Activity }\end{array} \\
\text { OR } \\
\end{array}$} & \\
\hline & OR & $95 \% \mathrm{CI}$ & OR & $95 \% \mathrm{CI}$ & OR & $95 \% \mathrm{CI}$ & OR & $95 \% \mathrm{CI}$ & OR & $95 \%$ CI & & \\
\hline \multicolumn{13}{|l|}{ Boys } \\
\hline \multicolumn{13}{|l|}{ Household income } \\
\hline High & 0.81 & $(0.78-0.84)$ & 0.80 & $(0.76-0.84)$ & 0.91 & $(0.87-0.95)$ & 1.08 & $(1.04-1.13)$ & 1.13 & $(1.09-1.18)$ & 1.00 & $<0.0001$ \\
\hline Medium-high & 0.75 & $(0.73-0.77)$ & 0.88 & $(0.85-0.90)$ & 0.87 & $(0.84-0.89)$ & 1.00 & $(0.97-1.02)$ & 1.00 & $(0.97-1.03)$ & 1.00 & $<0.0001$ \\
\hline Medium & 0.84 & $(0.82-0.86)$ & 0.88 & $(0.85-0.90)$ & 0.91 & $(0.89-0.93)$ & 0.97 & $(0.95-0.99)$ & 1.02 & $(1.00-1.04)$ & 1.00 & $<0.0001$ \\
\hline Medium-low & 0.76 & $(0.73-0.79)$ & 0.97 & $(0.92-1.02)$ & 0.91 & $(0.87-0.95)$ & 1.05 & $(1.01-1.09)$ & 1.07 & $(1.02-1.12)$ & 1.00 & $<0.0001$ \\
\hline Low & 0.51 & $(0.47-0.55)$ & 0.70 & $(0.63-0.78)$ & 0.83 & $(0.76-0.91)$ & 0.75 & $(0.69-0.81)$ & 0.93 & $(0.86-1.01)$ & 1.00 & $<0.0001$ \\
\hline Residence type & & & & & & & & & & & & $<0.0001$ \\
\hline Live with family & 0.80 & $(0.79-0.81)$ & 0.89 & $(0.87-0.90)$ & 0.90 & $(0.89-0.91)$ & 1.00 & $(0.99-1.01)$ & 1.04 & $(1.02-1.05)$ & 1.00 & $<0.0001$ \\
\hline Live with relative & 0.49 & $(0.42-0.57)$ & 0.36 & $(0.30-0.42)$ & 0.81 & $(0.69-0.95)$ & 0.89 & $(0.77-1.05)$ & 1.00 & $(0.85-1.17)$ & 1.00 & $<0.0001$ \\
\hline Accommodation, trace, dormitory & 0.74 & $(0.67-0.81)$ & 0.72 & $(0.65-0.81)$ & 0.65 & $(0.59-0.71)$ & 0.83 & $(0.76-0.92)$ & 0.95 & $(0.86-1.05)$ & 1.00 & 0.0027 \\
\hline Childcare facility & 0.49 & $(0.42-0.58)$ & 0.41 & $(0.33-0.51)$ & 0.96 & $(0.79-1.15)$ & 0.63 & $(0.53-0.76)$ & 0.55 & $(0.46-0.65)$ & 1.00 & 0.0366 \\
\hline \multicolumn{13}{|c|}{ Time spent sitting and studying on a weekday (hours) } \\
\hline$<8$ & 0.78 & $(0.77-0.80)$ & 0.89 & $(0.87-0.91)$ & 0.91 & $(0.89-0.93)$ & 1.00 & $(0.98-1.02)$ & 1.04 & $(1.02-1.07)$ & 1.00 & $<0.0001$ \\
\hline $8 \leq n<12$ & 0.79 & $(0.77-0.81)$ & 0.86 & $(0.83-0.89)$ & 0.87 & $(0.85-0.90)$ & 0.98 & $(0.95-1.01)$ & 1.00 & $(0.97-1.03)$ & 1.00 & $<0.0001$ \\
\hline $12 \leq n<16$ & 0.76 & $(0.73-0.78)$ & 0.79 & $(0.76-0.83)$ & 0.89 & $(0.86-0.92)$ & 0.97 & $(0.93-1.00)$ & 0.93 & $(0.90-0.97)$ & 1.00 & $<0.0001$ \\
\hline $16 \leq n<20$ & 0.84 & $(0.80-0.88)$ & 0.85 & $(0.80-0.89)$ & 0.86 & $(0.82-0.89)$ & 1.03 & $(0.99-1.08)$ & 1.20 & $(1.14-1.26)$ & 1.00 & $<0.0001$ \\
\hline \multicolumn{13}{|l|}{ Grade } \\
\hline High & 0.81 & $(0.78-0.84)$ & 0.82 & $(0.79-0.86)$ & 0.85 & $(0.82-0.88)$ & 1.10 & $(1.06-1.15)$ & 1.09 & $(1.05-1.14)$ & 1.00 & $<0.0001$ \\
\hline Medium-high & 0.78 & $(0.76-0.81)$ & 0.90 & $(0.87-0.93)$ & 0.89 & $(0.86-0.91)$ & 1.02 & (0.99-1.05) & 1.13 & $(1.10-1.17)$ & 1.00 & $<0.0001$ \\
\hline Medium & 0.76 & $(0.74-0.78)$ & 0.88 & $(0.85-0.91)$ & 0.90 & $(0.88-0.93)$ & 0.93 & $(0.90-0.95)$ & 1.01 & $(0.98-1.04)$ & 1.00 & $<0.0001$ \\
\hline Medium-low & 0.83 & $(0.81-0.86)$ & 0.96 & $(0.93-1.00)$ & 0.96 & $(0.94-0.99)$ & 1.00 & (0.98-1.03) & 1.00 & $(0.97-1.03)$ & 1.00 & $<0.0001$ \\
\hline Low & 0.74 & $(0.71-0.77)$ & 0.65 & $(0.61-0.68)$ & 0.83 & $(0.79-0.87)$ & 0.95 & $(0.91-0.99)$ & 0.89 & $(0.86-0.93)$ & 1.00 & $<0.0001$ \\
\hline \multicolumn{13}{|l|}{ Depression } \\
\hline Yes & 0.59 & $(0.56-0.63)$ & 0.68 & $(0.64-0.72)$ & 0.78 & $(0.74-0.83)$ & 0.85 & $(0.81-0.90)$ & 0.85 & $(0.80-0.90)$ & 1.00 & $<0.0001$ \\
\hline No & 0.81 & $(0.80-0.82)$ & 0.88 & $(0.87-0.90)$ & 0.90 & $(0.89-0.91)$ & 1.00 & $(0.99-1.02)$ & 1.04 & (1.03-1.06) & 1.00 & $<0.0001$ \\
\hline \multicolumn{13}{|l|}{ Suicidal ideation } \\
\hline Yes & 0.42 & $(0.39-0.46)$ & 0.54 & $(0.49-0.60)$ & 0.67 & $(0.61-0.74)$ & 0.77 & $(0.71-0.85)$ & 0.86 & $(0.78-0.95)$ & 1.00 & $<0.0001$ \\
\hline No & 0.81 & $(0.79-0.82)$ & 0.88 & $(0.86-0.89)$ & 0.90 & $(0.89-0.91)$ & 1.00 & $(0.98-1.01)$ & 1.03 & (1.02-1.05) & 1.00 & $<0.0001$ \\
\hline
\end{tabular}


Table 3. Cont.

\begin{tabular}{|c|c|c|c|c|c|c|c|c|c|c|c|c|}
\hline \multirow{3}{*}{ Variable } & \multicolumn{11}{|c|}{ Number of Days of Intense Physical Activity per Week } & \multirow{3}{*}{$\begin{array}{l}p \text {-Value } \\
\text { for Trend }\end{array}$} \\
\hline & \multicolumn{2}{|c|}{ More than 5 Days a Week } & \multicolumn{2}{|c|}{4 Days } & \multicolumn{2}{|c|}{3 Days } & \multicolumn{2}{|c|}{2 Days } & \multicolumn{2}{|c|}{1 Day } & \multirow{2}{*}{$\begin{array}{c}\text { Non-Physical } \\
\text { Activity }\end{array}$} & \\
\hline & OR & $95 \% \mathrm{CI}$ & OR & $95 \% \mathrm{CI}$ & OR & $95 \% \mathrm{CI}$ & OR & $95 \% \mathrm{CI}$ & OR & $95 \% \mathrm{CI}$ & & \\
\hline \multicolumn{13}{|l|}{ Girls } \\
\hline \multicolumn{13}{|l|}{ Household income } \\
\hline High & 0.59 & $(0.55-0.63)$ & 0.77 & $(0.72-0.83)$ & 0.81 & $(0.77-0.85)$ & 0.76 & $(0.73-0.80)$ & 0.81 & $(0.77-0.84)$ & 1.00 & $<0.0001$ \\
\hline Medium-high & 0.79 & $(0.76-0.83)$ & 0.92 & $(0.88-0.96)$ & 0.85 & $(0.83-0.88)$ & 0.92 & $(0.90-0.95)$ & 0.98 & $(0.95-1.00)$ & 1.00 & $<0.0001$ \\
\hline Medium & 0.80 & $(0.77-0.82)$ & 0.86 & $(0.83-0.90)$ & 0.81 & $(0.79-0.83)$ & 0.89 & $(0.87-0.91)$ & 0.93 & $(0.91-0.95)$ & 1.00 & $<0.0001$ \\
\hline Medium-low & 0.92 & $(0.86-0.99)$ & 0.76 & $(0.70-0.82)$ & 0.94 & $(0.89-1.00)$ & 1.08 & $(1.02-1.13)$ & 1.08 & $(1.03-1.13)$ & 1.00 & 0.0011 \\
\hline Low & 0.61 & $(0.53-0.71)$ & 0.37 & $(0.31-0.43)$ & 0.83 & $(0.72-0.96)$ & 0.98 & $(0.87-1.11)$ & 1.10 & $(0.98-1.23)$ & 1.00 & 0.0007 \\
\hline \multicolumn{13}{|l|}{ Residence type } \\
\hline Live with family & 0.80 & $(0.78-0.82)$ & 0.88 & $(0.86-0.90)$ & 0.85 & $(0.84-0.86)$ & 0.90 & $(0.89-0.92)$ & 0.95 & $(0.93-0.96)$ & 1.00 & $<0.0001$ \\
\hline Live with relative & 0.14 & $(0.11-0.19)$ & 0.12 & $(0.09-0.15)$ & 0.48 & $(0.37-0.62)$ & 1.16 & $(0.87-1.54)$ & 0.81 & $(0.64-1.03)$ & 1.00 & $<0.0001$ \\
\hline Accommodation, trace, dormitory & 0.65 & $(0.55-0.76)$ & 0.41 & $(0.35-0.48)$ & 0.48 & $(0.42-0.54)$ & 0.91 & $(0.81-1.02)$ & 1.07 & $(0.96-1.20)$ & 1.00 & 0.0006 \\
\hline Childcare facility & 0.12 & $(0.09-0.17)$ & 0.38 & $(0.27-0.54)$ & 0.62 & $(0.47-0.81)$ & 0.53 & $(0.40-0.69)$ & 0.63 & $(0.48-0.83)$ & 1.00 & $<0.0001$ \\
\hline \multicolumn{13}{|c|}{ Time spent sitting and studying on a weekday (hours) } \\
\hline$<8$ & 0.76 & $(0.73-0.79)$ & 0.85 & $(0.82-0.89)$ & 0.91 & $(0.88-0.94)$ & 0.92 & $(0.90-0.95)$ & 0.93 & $(0.91-0.95)$ & 1.00 & $<0.0001$ \\
\hline $8 \leq n<12$ & 0.93 & $(0.89-0.97)$ & 0.86 & $(0.82-0.91)$ & 0.84 & $(0.81-0.87)$ & 0.93 & $(0.90-0.95)$ & 1.01 & $(0.99-1.04)$ & 1.00 & $<0.0001$ \\
\hline $12 \leq n<16$ & 0.71 & $(0.68-0.74)$ & 0.81 & $(0.77-0.85)$ & 0.83 & $(0.81-0.86)$ & 0.84 & $(0.81-0.86)$ & 0.90 & $(0.87-0.92)$ & 1.00 & $<0.0001$ \\
\hline $16 \leq n<20$ & 0.63 & $(0.59-0.67)$ & 0.85 & $(0.80-0.91)$ & 0.68 & $(0.65-0.71)$ & 0.94 & $(0.90-0.97)$ & 0.96 & $(0.92-0.99)$ & 1.00 & $<0.0001$ \\
\hline \multicolumn{13}{|l|}{ Grade } \\
\hline High & 0.66 & $(0.62-0.70)$ & 0.73 & $(0.68-0.77)$ & 0.75 & $(0.71-0.78)$ & 0.89 & $(0.85-0.92)$ & 1.03 & $(0.99-1.08)$ & 1.00 & $<0.0001$ \\
\hline Medium-high & 0.65 & $(0.63-0.68)$ & 0.87 & $(0.83-0.91)$ & 0.90 & $(0.87-0.93)$ & 0.88 & $(0.86-0.91)$ & 0.92 & $(0.89-0.94)$ & 1.00 & $<0.0001$ \\
\hline Medium & 0.88 & $(0.84-0.92)$ & 1.03 & $(0.98-1.08)$ & 0.82 & $(0.80-0.85)$ & 0.96 & $(0.93-0.99)$ & 0.90 & $(0.88-0.93)$ & 1.00 & $<0.0001$ \\
\hline Medium-low & 0.82 & $(0.79-0.86)$ & 0.70 & $(0.66-0.74)$ & 0.76 & $(0.74-0.79)$ & 0.82 & $(0.79-0.85)$ & 0.93 & $(0.90-0.96)$ & 1.00 & $<0.0001$ \\
\hline Low & 0.90 & $(0.83-0.96)$ & 0.86 & $(0.78-0.94)$ & 1.09 & $(1.01-1.17)$ & 1.02 & $(0.96-1.08)$ & 1.16 & $(1.10-1.22)$ & 1.00 & 0.1736 \\
\hline \multicolumn{13}{|l|}{ Depression } \\
\hline Yes & 0.55 & $(0.51-0.58)$ & 0.55 & $(0.52-0.59)$ & 0.70 & $(0.66-0.74)$ & 0.83 & $(0.78-0.87)$ & 1.02 & $(0.97-1.07)$ & 1.00 & $<0.0001$ \\
\hline No & 0.81 & $(0.79-0.83)$ & 0.89 & $(0.87-0.92)$ & 0.85 & $(0.84-0.87)$ & 0.91 & $(0.90-0.93)$ & 0.94 & $(0.93-0.95)$ & 1.00 & $<0.0001$ \\
\hline \multicolumn{13}{|l|}{ Suicidal ideation } \\
\hline Yes & 0.57 & $(0.52-0.63)$ & 0.68 & $(0.61-0.77)$ & 0.76 & $(0.69-0.83)$ & 0.93 & $(0.85-1.01)$ & 1.06 & $(0.98-1.16)$ & 1.00 & $<0.0001$ \\
\hline No & 0.79 & $(0.77-0.80)$ & 0.86 & $(0.83-0.88)$ & 0.84 & $(0.83-0.86)$ & 0.90 & $(0.89-0.92)$ & 0.94 & $(0.93-0.96)$ & 1.00 & $<0.0001$ \\
\hline
\end{tabular}




\section{Discussion}

Our study was conducted to investigate the relationship between intense physical activity and stress in Korean adolescents. Our results indicated that about $78.9 \%$ of Korean youth felt stressed. We were able to confirm that the response rate of girls feeling stressed was higher than boys. However, both boys and girls were able to confirm that when they engaged in more intense physical activity, their response rate to stress was lower.

Some argue that the right amount of stress can further improve the lives of adults [23]. However, adolescence is subject to sudden physical changes and is vulnerable to various external factors; sustained stress during adolescence may have a negative impact, both physically and psychologically [24,25]. Therefore, in order for adolescents to overcome stress, it is necessary to understand the causes of stress and learn how to cope with them. There are numerous factors that cause stress in our lives, but there are also many ways to manage this stress [26-29]. Experts suggest that sleep therapy, diet, mental training, and physical activity are efficient ways to manage stress. In line with our research findings, according to a study involving 7-8 year old girls in the U.S. conducted by the American Psychological Association, exercise is effective in stress management [30]. The positive effects of exercise on stress have been proven both in quantitative and qualitative studies [31,32]. Studies conducted in Thailand showed that the discharge of cortisol and epinephrine, which are stress-inducing hormones, was decreased for $24 \mathrm{~h}$ by jogging five times per week [33-35].

Through this study, we were able to identify the trends associated with stress in Korean adolescents. First, the stress experienced by Korean students should no longer be regarded as an issue for students to manage themselves. In our study, $78.9 \%$ of Korean students answered that they are under stress. Of all the respondents, $73.2 \%$ of boys and $84.5 \%$ of girls answered that they are under stress. This means that a large number of students are exposed to factors that cause stress. Further, stress causes physical, psychological, and mental diseases. Our study also found that stress was related to depression or suicidal ideation. Therefore, we should not demand that adolescents address their stress themselves. This suggests that governments, academia, schools, and families need to take measures to effectively manage stress. Second, regular intense physical activity may be effective in adolescent stress management. Students, both boys and girls, who engaged in intense physical activity for three days or more each week were less stressed than those who did not. However, the stress experienced by people who exercise less than twice per week is similar to those who do not exercise at all. This may mean that a certain frequency of physical activity causes a physiological mechanism to reduce stress. Despite the apparent effectiveness of physical activity, some students may not be able to engage in physical activity due to a lack of time. Therefore, we propose that schools guarantee that physical education is provided at least three times per week for the effective stress management of Korean adolescents. Third, girls are more vulnerable to stress than boys. About $73 \%$ of the boys responded that they felt stressed, compared to about $85 \%$ of the girls. It is also possible that girls are more stressed than boys because of cultural expectations and gender-stereotypical behaviors [17]. However, considering the girls' stress response rate is $85 \%$, it is need a stress management system for girls. Therefore, as an alternative, we believe that developing an exercise program specifically for girls can help to effectively manage their stress. These observations are in line with the guidelines of the World Health Organization and American College of Sports Medicine [36,37]. Therefore, while it is best to engage in intense physical activity as much as possible, when considering busy students' schedules, it is necessary to examine ways to institutionalize physical activity so that students can exercise at least three times per week.

However, our study has some limitations. First of all, since this was a cross-sectional study, a causal relationship between intense physical activity and stress could not be established. Second, the KYRBWS questions investigate the average of the past year. The data used in this study were mostly based on self-reported surveys; hence, questions on socioeconomic status, health behavior, and the intense physical activity covariate may have been subjected to recall bias. Third, the measurement of stress depends on self-diagnosis, and qualitative research through experimental testing is necessary for the accurate measurement of stress. Fourth, the effect of intense physical activity on stress was 
obvious. However, the effect was not significant. The stress experienced by students who engaged in intense physical activity for three days did not differ greatly from those who engaged in intense physical activity for five days or more. Nevertheless, students with no physical activity, once-weekly intense physical activity, or twice-weekly intense physical activity were about 1.3 times more stressed.

Despite these limitations, our research has several strengths. First, this study used primary data suitable for the study of Korean adolescents. These data are sufficient to represent the phenomenon for Korean adolescents, since 170,000 respondents were included. Second, we have accumulated six years of data to study the phenomenon that occurs in adolescents. Therefore, the correlation between stress and intense physical activity in adolescents is reliable. Third, it provides information on the appropriate level of intense physical activity required for Korean adolescents to manage stress. Our study showed that intense physical activity in each assay was not significantly different from more than five days per week when it was more than three times per week. However, students who engaged in intense physical activity less than two times per week were more stressed. Therefore, when establishing a system and policy for the mental health management of adolescents, the results of this study could be utilized; intense physical activity three times per week could be made mandatory in class or club activities.

\section{Conclusions}

Our study identified that intense physical activity in Korean adolescents is associated with a decrease in stress. In addition, the least amount of stress was found in adolescents who engaged in frequent intense physical activity. Therefore, we propose to establish a system to guarantee physical activity time for the health of Korean adolescents. If intense physical activity is difficult to perform on a daily basis, we suggest engaging in intense physical activity at least three times per week.

Author Contributions: Conceptualization, H.J.K. and J.K.; Formal analysis, H.J.K. and S.Y.O.; Methodology, H.J.K. and D.W.L.; Supervision, E.-C.P.; Writing—original draft, H.J.K.; Writing—review \& editing, E.-C.P.

Funding: This research received no external funding.

Acknowledgments: We are grateful to the Korea Centers for Disease Control and Prevention (KCDC) that conducted the Korea Youth Risk Behavior Web-based Survey which is the primary source of our study.

Conflicts of Interest: The authors declare no conflict of interest.

\section{References}

1. Jones, T.L. Definition of stress. In Eating Disorders in Women and Children: Prevention, Stress Management, and Treatment; CRC Press: Boca Raton, FL, USA, 2001; pp. 89-100.

2. Levine, S. A definition of stress? In Animal Stress; Springer: New York, NY, USA, 1985; pp. 51-69.

3. Ulrich-Lai, Y.M.; Herman, J.P. Neural regulation of endocrine and autonomic stress responses. Nat. Rev. Neurosci. 2009, 10, 397-409. [CrossRef] [PubMed]

4. Walker, E.F.; Walder, D.J.; Reynolds, F. Developmental changes in cortisol secretion in normal and at-risk youth. Dev. Psychopathol. 2001, 13, 721-732. [CrossRef]

5. Stephens, M.A.C.; Wand, G. Stress and the hpa axis: Role of glucocorticoids in alcohol dependence. Alcohol Res. 2012, 34, 468-483.

6. Tanner, J.M. Growth at Adolescence, 2nd ed.; Thomas: Springfield, IN, USA, 1962.

7. Byrne, B. Relationship between anxiety, fear, self-esteem, and coping strategies in adolescence. Adolescence 2000, 35, 201-215. [PubMed]

8. Blos, P. The second individuation process of adolescence. Psychoanal. Study Child 1967, 22, 162-186. [CrossRef]

9. MacPhee, A.R.; Andrews, J.W. Risk factors for depression in early adolescence. Adolescence 2006, 41, 435-466. [PubMed]

10. Larson, R.; Ham, M. Stress and "storm and stress" in early adolescence: The relationship of negative events with dysphoric affect. Dev. Psychol. 1993, 29, 130-140. [CrossRef]

11. Statistics Korea. 2018 Youth Statistics; KOSIS: Daejeon, Korea, 2018. 
12. Foa, E.B.; Constance, V.; Hembree, E.A.; Jaycox, L.H.; Meadows, E.A.; Street, G.P. A comparison of exposure therapy, stress inoculation training, and their combination for reducing posttraumatic stress disorder in female assault victims. Am. Psychol. Assoc. 1999, 67, 194-200. [CrossRef]

13. Ceriello, A. New insights on oxidative stress and diabetic complications may lead to a "causal" antioxidant therapy. Diabetes Care 2003, 26, 1589-1596. [CrossRef]

14. Quick, J.C.; Wright, T.A.; Adkins, J.A.; Nelson, D.L.; Quick, J.D. Preventive Stress Management in Organizations, 2nd ed.; American Psychological Association: Washington, DC, USA, 2013.

15. Murphy, L.R. Stress management in work settings: A critical review of the health effects. Am. J. Health Promot. 1996, 11, 112-135. [CrossRef]

16. Colten, M. Adolescent Stress: Causes and Consequences; Routledge: New York, NY, USA, 2017.

17. Dhull, I.; Kumari, S. Academic stress among adolescent in relation to gender. Int. J. Appl. Res. 2015, 1, $394-396$.

18. Shephard, R.J. Physical activity, fitness, and health: The current consensus. Quest 1995, 47, $288-303$. [CrossRef]

19. Bandura, A. Health promotion from the perspective of social cognitive theory. Psychol. Health 1998, 13, 623-649. [CrossRef]

20. Korea Sports Promotion Foundation. National Fitness Award 100; KSPF: Seoul, Korea, 2012.

21. Kim, Y.S.; Bae, C.W. The effects of regular walking exercise and jogging exercise on plasma $\beta$-endorphin, prolactin, cortisol. Korea Sport Res. 2003, 14, 545-554.

22. Cho, K.S.; Yoo, Y.C.; Jeon, Y.J. Movement of electoroencephalogram and plasm $\beta$-endorphin in the aerobic exercise. Korea Sport Res. 2003, 14, 717-725.

23. Suo, L.; Zhao, L.; Si, J.; Liu, J.; Zhu, W.; Chai, B.; Zhang, Y.; Feng, J.; Ding, Z.; Luo, Y.; et al. Predictable chronic mild stress in adolescence increases resilience in adulthood. Neuropsychopharmacology 2013, 38, 1387. [CrossRef]

24. Greene, J.W.; Walker, L.S. Psychosomatic problems and stress in adolescence. Pediatr. Clin. North Am. 1997, 44, 1557-1572. [CrossRef]

25. Bernhard, A.; Van der Merwe, C.; Ackermann, K.; Martinelli, A.; Neumann, I.D.; Freitag, C.M. Adolescent oxytocin response to stress and its behavioral and endocrine correlates. Horm. Behav. 2018, 105, 157-165. [CrossRef] [PubMed]

26. Straus, M.A. Social stress and marital violence in a national sample of american families. In Physical Violence in American Families; Routledge: New York, NY, USA, 2017; pp. 181-202.

27. Lee, J.Y.; Kim, J.H.; Cho, H.R.; Lee, J.S.; Ryu, J.M.; Yum, M.S.; Ko, T.S. Children experiencing first-time or prolonged febrile seizure are prone to stress hyperglycemia. J. Child Neurol. 2016, 31, 439-443. [CrossRef]

28. Grekin, R.; O'Hara, M.W. Prevalence and risk factors of postpartum posttraumatic stress disorder: A meta-analysis. Clin. Psychol. Rev. 2014, 34, 389-401. [CrossRef]

29. Chauvet, G.; Jean, C.; Bonin, B. Stress, anxiety and depression in heart disease patients: A major challenge for cardiac rehabilitation. Ann. Phys. Rehabil. Med. 2017, 60, 6-12. [CrossRef] [PubMed]

30. Brown, J.D.; Siegel, J.M. Exercise as a buffer of life stress: Aprospective study of adolescent health. Am. Psychol. Assoc. 2016, 7, 341-353. [CrossRef]

31. Norris, R.; Carroll, D.; Cochrane, R. The effects of physical activity and exercise training on psychological stress and well-being in an adolescent population. J. Psychosom. Res. 1992, 36, 55-65. [CrossRef]

32. Santos, S.A.; Rebelo, M.I.; Castro, E.M.B.; Belo, L.; Guerra, A.; Rego, C.; Quintanilha, A. Leukocyte activation, erythrocyte damage, lipid profile and oxidative stress imposed by high competition physical exercise in adolescents. Clin. Chim. Acta 2001, 306, 119-126. [CrossRef]

33. Nabkasorn, C.; Miyai, N.; Sootmongkol, A.; Junprasert, S.; Yamamoto, H.; Arita, M.; Miyashita, K. Effects of physical exercise on depression, neuroendocrine stress hormones and physiological fitness in adolescent females with depressive symptoms. Eur. J. Public Health 2006, 16, 179-184. [CrossRef]

34. Fevolden, S.E.; Røed, K.H.; Gjerde, B. Genetic components of post-stress cortisol and lysozyme activity in atlantic salmon; correlations to disease resistance. Fish Shellfish Immunol. 1994, 4, 507-519. [CrossRef]

35. Baker, G.H.; Irani, M.S.; Byrom, N.A.; Nagvekar, N.M.; Wood, R.J.; Hobbs, J.R.; Brewerton, D.A. Stress, cortisol concentrations, and lymphocyte subpopulations. Br. Med. J. (Clin. Res. Ed.) 1985, 290, 1393. [CrossRef] 
36. World Health Oraganization. Physical Activity; WHO: Geneva, Switzerland, 2018.

37. Balady, G.J. Acsm's Guidelines for Exercise Testing and Prescription, 6th ed.; ACSM: Indianapolis, IN, USA, 2000.

(C) 2019 by the authors. Licensee MDPI, Basel, Switzerland. This article is an open access article distributed under the terms and conditions of the Creative Commons Attribution (CC BY) license (http://creativecommons.org/licenses/by/4.0/). 